Copyright by the Acoustical Society of America. Clark, R. L., \& Gibbs, G. P. (1994). A novel approach to feedforward higher-harmonic control. Journal of the

Acoustical Society of America, 96(2), 926-936. doi: 10.1121/1.411389

\title{
A novel approach to feedforward higher-harmonic control
}

\author{
Robert L. Clark \\ Department of Mechanical Engineering and Materials Science, Duke University, Durham, North Carolina \\ 27708-0300
}

Gary P. Gibbs

Department of Mechanical Engineering, Virginia Polytechnic Institute and State University, Blacksburg, Virginia 24061

(Received 6 July 1993; revised 21 March 1994; accepted 22 April 1994)

\begin{abstract}
A novel adaptive filter structure is proposed for the control of systems characterized by higher harmonic response. The control approach has been designated the higher harmonic least-mean squares (HLMS) algorithm to differentiate it from the standard multifrequency filtered-x version of the least-mean squares approach. In the HLMS algorithm, a single frequency reference at that of the fundamental is all that is required to implement the controller. The remaining harmonics are generated internally based upon simple trigonometric relationships. The filtered-x LMS algorithm is implemented in parallel for each frequency to be controlled, minimizing or totally eliminating the contribution of the time-varying terms during the convergence process and increasing the rate of convergence for the higher harmonic control application. Results from the simulation demonstrate that the HLMS approach is far superior to the standard multifrequency, filtered-x LMS algorithm in adaptive, feedforward, higher-harmonic control.
\end{abstract}

PACS numbers: $43.40 . \mathrm{Vn}$

\section{INTRODUCTION}

Higher-harmonic control has been a subject of interest for years in the helicopter industry because of noise and vibration problems resulting from rotor dynamics. A number of papers have discussed and outlined procedures for controlling the vibration levels in such systems based on modelinsensitive approaches (Shaw and Albion, 1981; Shaw et al., 1989). In fact, Hall and Wereley (1989) detailed the specifics of an analog higher-harmonic control algorithm based upon Shaw's discrete time implementation that yields an identical compensator transfer function to that of the least mean squares (LMS) analog compensator. Sievers and von Flotow (1990) discuss and compare the two compensator designs. However, implementation of the multifrequency, filtered-x LMS algorithm for higher-harmonic control is complicated by time-varying terms present in the adaptation process. Glover (1977) discussed the implications of the time-varying terms and demonstrated that in the case of adaptive signal processing, the algorithm typically converges to a dynamic solution which is inconsistent with the optimal Wiener-Hopf solution. For the single frequency control problem, Elliot et al. (1987) observed that synchronous sampling of the reference signal resulted in a linear, time-invariant adaptation process. While this enhances the rate of convergence with the filtered-x, LMS algorithm, it is not a requirement for convergence. However, when a multiple frequency reference signal is required, the contribution of the time-varying terms must be minimized if the optimal Wiener-Hopf solution for the filter is desired.

The focus of this work is to outline a novel filter structure for the active control of higher-harmonic response of linear systems which overcomes problems associated with time-varying terms in the adaptation of the filter. A procedure is outlined whereby the harmonics of the fundamental are synthesized from simple trigonometric relationships to generate independent reference signals for each frequency to be controlled. The advantage to this approach is that a separate adaptive finite impulse response (FIR) filter can be constructed for each frequency such that a linear, time-invariant (LTI) solution, which results in the optimal Wiener-Hopf solution for each parallel filter structure, can be obtained. Glover (1977) eluded to the advantages of implementing multiple filters in parallel but noted that independent reference signals "that included every sinusoidal interference" must be available. An example problem was presented in his work whereby a sinusoidal reference was clipped to yield a square wave at the same frequency to generate the odd harmonics. However, the harmonics were superposed and thus a 64-coefficient FIR filter was implemented to effectively place a notch at each of the three harmonic reference signals desired for the active noise control (ANC) example presented.

With the filter structure and method of generating the harmonics in software proposed in this work, three FIR filters with two coefficients per filter are all that is required to achieve optimal system performance in the example provided by Glover (1977). Thus, the approach outlined reduces the number of adaptive filter coefficients required from 64 to 6 for that particular example. If the algorithm is implemented on a single digital signal processor, the sampling rate should be set at a frequency four times greater than that of the highest harmonic to be controlled. When standard oversampling techniques are employed, each parallel FIR filter can be implemented such that the time-varying (TV) portion is minimized or reduced to zero, increasing the overall rate and stability of convergence.

Elliot et al. (1987) demonstrated that for synchronous 


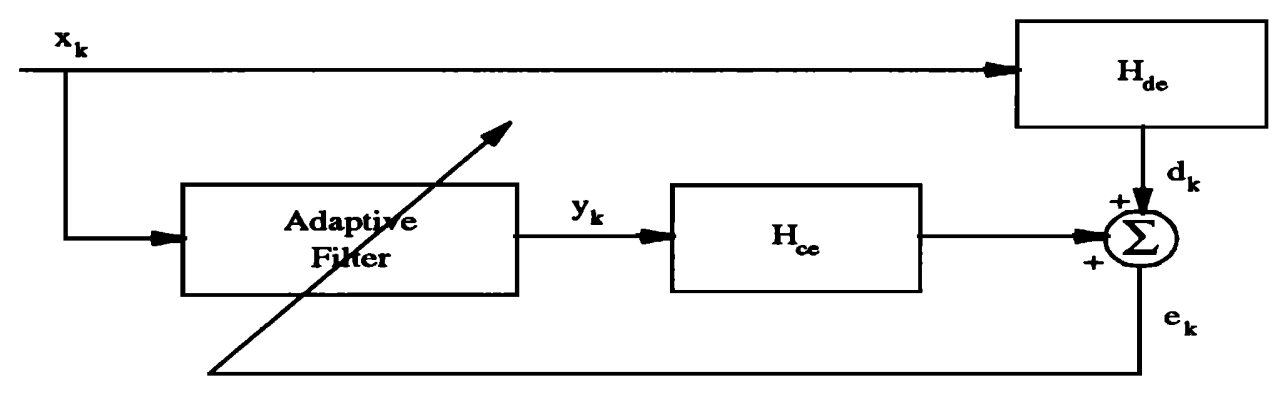

FIG. 1. Schematic of SISO adaptive filter for structural control.

sampling of a single-frequency reference, a linear timeinvariant (LTI) system results when the order of the filter is an integer multiple of half the number of samples per cycle. Thus, for a two-coefficient FIR filter, four samples per period is required to eliminate the TV contribution during the adaptation process for a single-frequency disturbance. Clark and Gibbs (1994) previously demonstrated that sampling at a rate of four samples per cycle yields optimal performance in adaptive noise cancellation of a single harmonic since the input correlation matrix is diagonal, and the minimum meansquare error (MSE) is achievable. A diagonal input correlation matrix simply indicates that the adaptation of the filter coefficients takes place on the principle axes of the paraboloid and hence each of the FIR filter coefficients is adapted independently. The eigenvalues of the input correlation matrix dictate the maximum value of the convergence parameter which can be used, and in the ideal case, the input correlation matrix is the identity matrix. Sievers and von Flotow (1990) suggested a modification to the filtered-x, multi-input/multioutput (MIMO), LMS algorithm proposed by Elliot et al. (1987) to incorporate this feature. Thus, a diagonal input correlation matrix provides significant performance advantages over a nondiagonal matrix both for adaptive interference cancellation and in adaptive feedforward control of linear systems.

To demonstrate the advantages of the proposed system, a simulation is performed on a simply supported beam. A single-input/single-output (SISO) system is considered and the multifrequency, filtered-x LMS algorithm is compared to the HLMS algorithm to demonstrate the performance advantages of the latter. Results from this work show that the proposed filter structure out performs the standard adaptive FIR filter structure in efficiency, rate of convergence, and minimum mean-squared error achievable.

\section{REVIEW OF STANDARD MULTIFREQUENCY LMS CONTROL}

Glover (1977) outlined a procedure for eliminating sinusoidal or other periodic interference corrupting a signal for applications in adaptive signal processing. Elliot et al. (1987) extended this work to include the active control of linear systems with multiple inputs and outputs. Elliot et al. (1987) also observed that for synchronous sampling of a singlefrequency reference signal, if the number of adaptive filter coefficients are an integer multiple of half the number of samples per cycle, then the adaptation of the filter is LTI. In this section, the discussion of the LTI and TV terms present in the adaptation process is extended to include a multifrequency reference input. A schematic diagram of a simple SISO, linear system is illustrated in Fig. 1 where an adaptive filter is implemented to provide the optimal control signal for minimizing the response of the disturbance at the chosen error signal. The system transfer function between the disturbance and the error is denoted by $H_{d e}$, and the transfer function between the control actuator and the error is represented by $H_{c e}$. A reference input correlated with the disturbance input is fed to an adaptive FIR filter to generate the optimal control signal through the control-error path.

The reference input is assumed to be multifrequency such that

$$
x(k)=\sum_{m=1}^{M} \cos (\omega m k T),
$$

where $M$ is the number of frequencies present, $\omega$ is the circular frequency of the reference input, $m$ represents the $(m-1)$ th harmonic of that reference, $k$ is the time step, and $T$ is the sample period. The filtered-x LMS algorithm can be expressed as follows for the given reference input (Widrow and Stearns, 1985):

$$
w_{i}(k+1)=w_{i}(k)-\mu e(k) \hat{x}(k-i),
$$

where $w_{i}(k)$ is the $i$ th coefficient of the adaptive filter at the $k$ th time step, $\mu$ is the convergence parameter, and $\hat{x}(k)$ is the filtered-x signal at the $k$ th time step. If the modulus and phase of the transfer function $H_{c e}$ at each of the $(m-1)$ th harmonics is represented as

$$
H_{c e}(\exp (j \omega m T))=H_{m} \exp \left(j \Phi_{m}\right),
$$

where $H_{m}$ is the magnitude of the response at the $m$ th frequency and $\Phi_{m}$ is the phase of the response at the $m$ th frequency, then

$$
\hat{x}(k)=\sum_{m=1}^{M} H_{m} \cos \left(\omega m k T+\Phi_{m}\right) .
$$

Following the derivation in the Appendix, one can show that the $z$ transform of the output of the adaptive FIR filter can be expressed as a function of LTI and TV terms: 


$$
\begin{aligned}
Y(z)= & -\frac{\mu I}{4} \sum_{m=1}^{M} H_{m} E(z)[U(z \exp (-j \omega m T)) \\
& \left.\times \exp \left(-j \Phi_{m}\right)+U(z \exp (j \omega m T)) \exp \left(j \Phi_{m}\right)\right] \\
& -\frac{\mu}{4} \sum_{m=1}^{M} \sum_{n=1}^{M} H_{m} \beta\left(\frac{\omega(m-n)}{2} T, I\right)[\mathrm{TV}]_{1} \\
& -\frac{\mu}{4} \sum_{m=1}^{M} \sum_{n=1}^{M} H_{m} \beta\left(\frac{\omega(m+n)}{2} T, I\right)[\mathrm{TV}]_{2} \\
& m \neq n,
\end{aligned}
$$

where $Y(z)$ is the $z$ transform of the output of the adaptive filter, $E(z)$ is the $z$ transform of the error, $U(z)$ is $1 /(z-1)$, $I$ is the number of coefficients used in the adaptive filter, [TV] is used to represent the time-varying terms, and the function $\beta(\omega(m \pm n) T / 2, I)$ is expressed as follows:

$$
\beta\left(\frac{\omega(m \pm n)}{2} T, I\right)=\frac{\sin (\omega(m \pm n) T I / 2)}{\sin (\omega(m \pm n) T / 2)} .
$$

Thus if

$$
\frac{\omega(m \pm n) T I}{2}=p \pi
$$

where $p$ is some integer, $\beta(\omega(m \pm n) T / 2, I)=0$, and the system is LTI. However, for the general multifrequency control case, the TV terms cannot be totally eliminated. Thus, the order of the adaptive filter $(I)$ must be increased to reduce the contribution of the TV terms in Eq. (5). (Note that $I$ multiplies the LTI terms of the equation.) As Glover (1977) demonstrated in his example problem, increasing the order of the adaptive filter sharpens the notches at each of the reference frequencies. In theory, only two coefficients per frequency present in the reference are required to control the system response at the error if the system is controllable. However, because of the TV terms, the ratio of $\beta(\omega(m)$ $\pm n) T / 2, I) / I$ must be minimized [i.e., either $\beta(\omega(m \pm n) T /$ $2, I)=0$ or $I \gg \beta(\omega(m \pm n) T / 2, I)]$ to reduce the contribution of the TV terms. Otherwise, the system will converge to a dynamic solution, which is inconsistent with the optimal Wiener-Hopf solution.

To circumvent this problem, a novel filter structure is proposed for higher-harmonic control when the higher harmonics can be synthesized from the fundamental. Thus an LTI system results for each frequency to be control since a separate reference signal is available for independent adaptive FIR filters to generate the control input to the system. While the control approach outlined is implemented in the time domain, it is analogous to a frequency domain control approach since each frequency is independently controlled. The system is proposed for rapid and stable convergence and is outlined in the following section.

\section{OVERVIEW OF THE CONTROL APPROACH}

A schematic diagram of the higher-harmonic, leastmean-squares (HLMS) feedforward control algorithm and filter structure is presented in Fig. 2, and as indicated, up to
$N$ harmonics of the fundamental frequency can be included. The only measurable reference input is the single-frequency excitation driving the disturbance. The disturbance is assumed to generate harmonics of the fundamental reference, while the system response to the higher-harmonic input is assumed linear. Thus to effectively control the system with an array of control actuators and error sensors, the control input must consist of the fundamental and associated harmonics as well. Using simple trigonometric expressions, each harmonic can be expressed as a function of the fundamental reference. For example, if

$$
x_{0}(k)=\sin (2 \pi f k T),
$$

then

$$
\begin{aligned}
& x_{1}(k)=2 \times\left[x_{0}(k)\right]^{2}-1, \\
& x_{2}(k)=3 \times x_{0}(k)-4 \times\left[x_{0}(k)\right]^{3}, \\
& x_{3}(k)=2 \times\left[x_{1}(k)\right]^{2}-1, \\
& x_{4}(k)=5 \times x_{2}(k)-10 \times x_{0}(k)+16 \times\left[x_{0}(k)\right]^{4}
\end{aligned}
$$

and

$$
x_{5}(k)=2 \times\left[x_{2}(k)\right]^{2}-1,
$$

where $x_{0}(k)$ is the fundamental reference and $x_{s}(k)$ is the $s$ th associated harmonic at each time step $k$. The control is implemented at a frequency of $1 / T$, and the first five harmonics of the fundamental are shown here. Depending on the nature of the system response and level of the input disturbance, more or fewer harmonics can be included as is necessary.

Once the necessary reference signals for the harmonics of the fundamental are created, the HLMS controller can be implemented. The HLMS algorithm is unique in that the fundamental frequency and each respective harmonic are controlled independently, and the respective harmonics are generated internal to the algorithm. However, the control algorithm is based upon the filtered-x version of the adaptive LMS algorithm previously outlined by Widrow and Stearns (1985), as well as Elliot et al. (1987) for physical systems. The difference in the method of implementation chosen here lies in the parallel configuration of the algorithm for the fundamental reference and each associated harmonic. Thus the convergence parameter can be adjusted independently in each parallel implementation of the algorithm, increasing the rate of convergence irrespective of the impulse response of the system as discussed by Sievers and von Flotow (1990). Each adaptive filter simply consists of a two-coefficient finite-impulse response filter, which is all that is required to control each independent frequency.

The error for the multi-input/multi-output filtered-x HLMS algorithm is formulated as a function of the disturbance and the control as follows:

$$
\begin{aligned}
e_{l}(n)= & e_{l}^{D}(n)+\sum_{s=0}^{N_{h}} \sum_{m=1}^{M} \sum_{j=0}^{N_{p}-1} P_{s l m j} \\
& \times \sum_{i=0}^{N_{w}-1} w_{s m i}(n-j) x_{s}(n-i-j),
\end{aligned}
$$




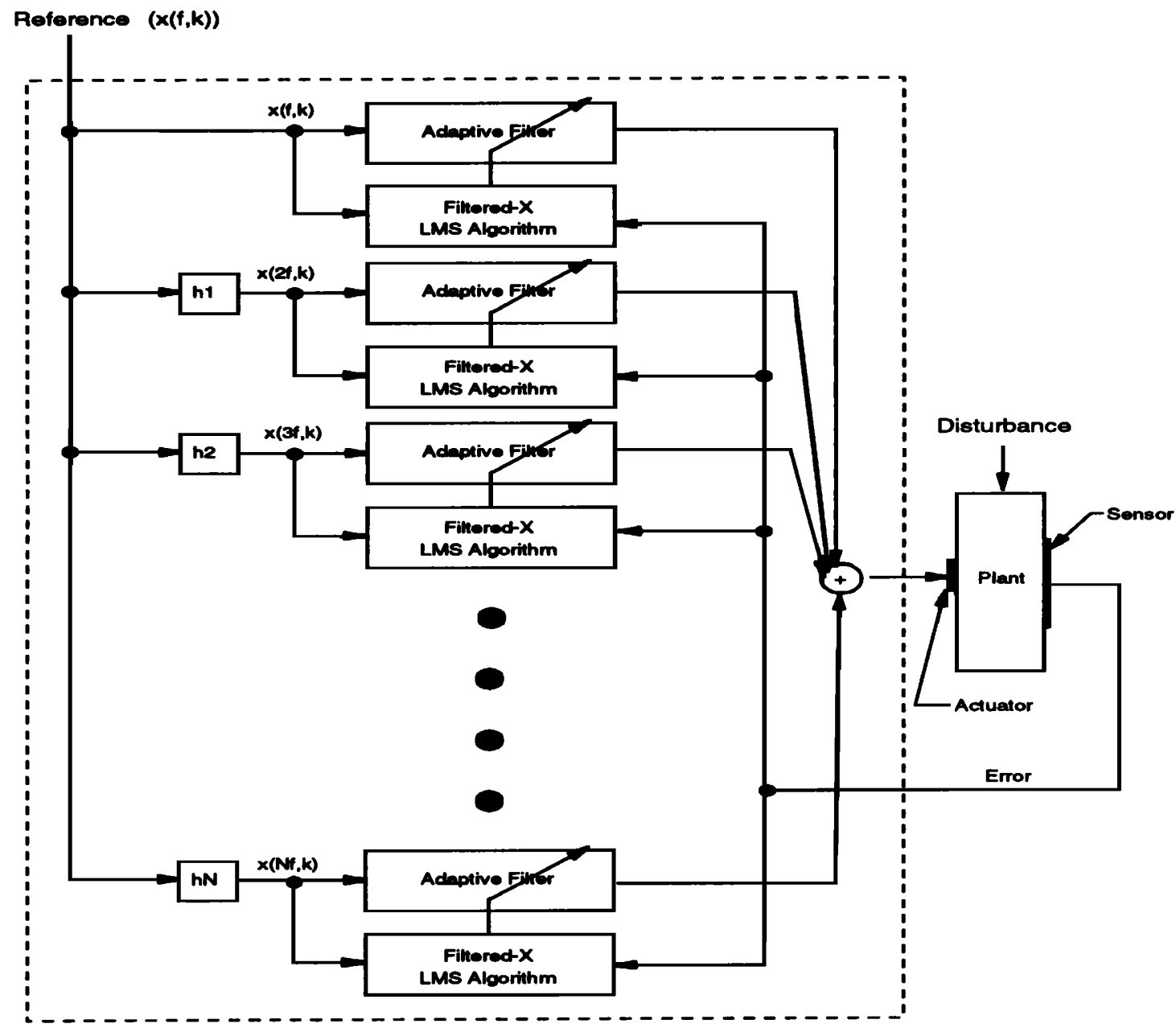

FIG. 2. Schematic of higher-harmonic control least-mean squares (HLMS) algorithm.

where $n$ is the time step number, $s$ is the subscript for harmonic number with zero being the fundamental, $m$ is the subscript for actuator number, $l$ is the subscript for error sensor number, $i$ is the subscript for adaptive filter coefficient number, $j$ is the subscript for fixed filter coefficient number, $e_{l}^{D}(n)$ is the error due to the disturbance at the $l$ th error sensor, $x(n)$ is the input reference source, $w_{s m i}$ is the coefficients of the adaptive finite impulse response (FIR) filter for the $m$ th actuator and the $i$ th coefficient at the sth harmonic, $\boldsymbol{P}_{\text {slmj }}$ is the $j$ th coefficient of the fixed (FIR) filter between the output of the $m$ th actuator and the $l$ th error sensor of the $s$ th harmonic, $M$ is the number of control actuators, $N_{p}$ is the number of fixed filter coefficients, $N_{w}$ is the number of adaptive filter coefficients, and $N_{h}$ is the number of harmonics included.

The signal chosen to be minimized is the mean-squared error defined by

$$
J=\mathrm{E}\left[\sum_{l=1}^{L} e_{l}^{2}(n)\right]
$$

where $\mathrm{E}[*]$ is the expectation operator, and $L$ is the number of error sensors. As the portion of the system to be controlled is linear, the function is quadratic with respect to the adaptive filter coefficients, resulting in a single minimum. The dynam- ics of the physical system to be controlled are modeled with the fixed filter coefficients $P_{s l m j}$ such that the adaptive filter coefficients can be appropriately updated as follows:

$$
w_{s m i}(n+1)=w_{s m i}(n)-\mu_{s} \sum_{l=1}^{L} e_{l}(n) r_{s l m}(n-i),
$$

where

$$
r_{s l m}(n-i)=\sum_{j=0}^{N_{p}-1} P_{s l m j} x_{s}(n-i-j),
$$

$x_{s}(n-i-j)$ is the reference at the sth harmonic, $r_{s l m}(n-i)$ is the filtered-x signal, and $\mu_{s}$ is the convergence parameter. The fixed finite-impulse response filters for each harmonic frequency are obtained from the standard implementation of the LMS algorithm. The specific harmonic reference signal is echoed through the control output channel, and the response at each of the chosen error sensors is sampled. The filter coefficients $\boldsymbol{P}_{\text {slm } j}$ between the $\boldsymbol{m}$ th output and the $l$ th error sensor of the sth harmonic are adapted such that when multiplied by the reference, the difference between the output of the filters and the selected error sensor is mathematically zero. Hence the LMS algorithm is used to perform the system identification between each channel of control and error 
sensor at each respective harmonic. Since the system identification is obtained for each harmonic independently, the full dynamic range of the analog to digital converters can be utilized in practice to increase the accuracy of the system identification at each harmonic in contrast to the typical multifrequency system identification procedure, which is dominated by the harmonics having the greatest amplitude in the system response.

\section{PRACTICAL CONSIDERATIONS}

For practical implementation of the control algorithm, one must address the throughput of the system as well as the convergence characteristics of the algorithm. For typical implementation of the filtered-x LMS algorithm (i.e., singlefrequency control) the sample rate is dictated by the frequency to be controlled. For example, if the system to be controlled is being forced at an excitation frequency of 200 $\mathrm{Hz}$, the signal must be sampled faster than $400 \mathrm{~Hz}$, (i.e., twice the excitation frequency) to prevent aliasing. However, an upper limit on the sampling rate is typically dictated by the time required to update the adaptive filter coefficients in the control algorithm (i.e., control system throughput). The question thus arises as to what the optimal sampling rate should be for a given excitation frequency. If the reference signal is synchronously sampled at four times per cycle for a single harmonic input, the two coefficients of an adaptive FIR filter are updated independently because of the orthogonality observed at the discrete sampling interval between $x(k)$ and $x(k-1)$. In other words, there is a perfect $90^{\circ}$ phase shift between the reference signal at time steps $x(k)$ and $x(k-1)$, respectively. (As discussed in Sec. I, this also corresponds to an LTI system.)

To provide an example, consider the discrete Fourier transform of a two-coefficient FIR filter:

$$
\begin{aligned}
W(\exp (j \omega T))= & \left(w_{0}+w_{1} \cos (2 \pi / N)\right) \\
& -j\left(w_{1} \sin (2 \pi / N)\right) .
\end{aligned}
$$

Upon studying this expression, one observes that as $N$ approaches 2, the Nyquist rate $w_{1}$ must approach infinity to represent any phase delay required in the signal and thus $w_{0}$ must also approach infinity. If $N=4$ is chosen, then the real part is represented totally by $w_{0}$ and the imaginary part is represented by $w_{1}$, decoupling the two coefficients. As $N$ approaches infinity, $w_{1}$ again must approach infinity to represent phase delays and $w_{0}$ must correspondingly do the same.

The off-diagonal terms of the input correlation matrix are identically zero when $N=4$, but they increase as $N \rightarrow 2$ or $N \rightarrow \infty$ for a harmonic signal. Thus, for all $N \neq 4$ the disparity in the eigenvalues of the input correlation matrix increases, and, as outlined by Griffiths (1975),

$$
\frac{-1}{\ln \left(1-\mu \lambda_{\max }\right)} \leqslant \tau_{a} \leqslant \frac{-1}{\ln \left(1-\mu \lambda_{\min }\right)},
$$

where $\tau_{a}$ is the overall time constant of the algorithm, $\mu$ is the convergence parameter, and $\lambda_{\min }$ and $\lambda_{\max }$ are the minimum and maximum eigenvalues of the input correlation matrix. When the input correlation matrix is diagonal,

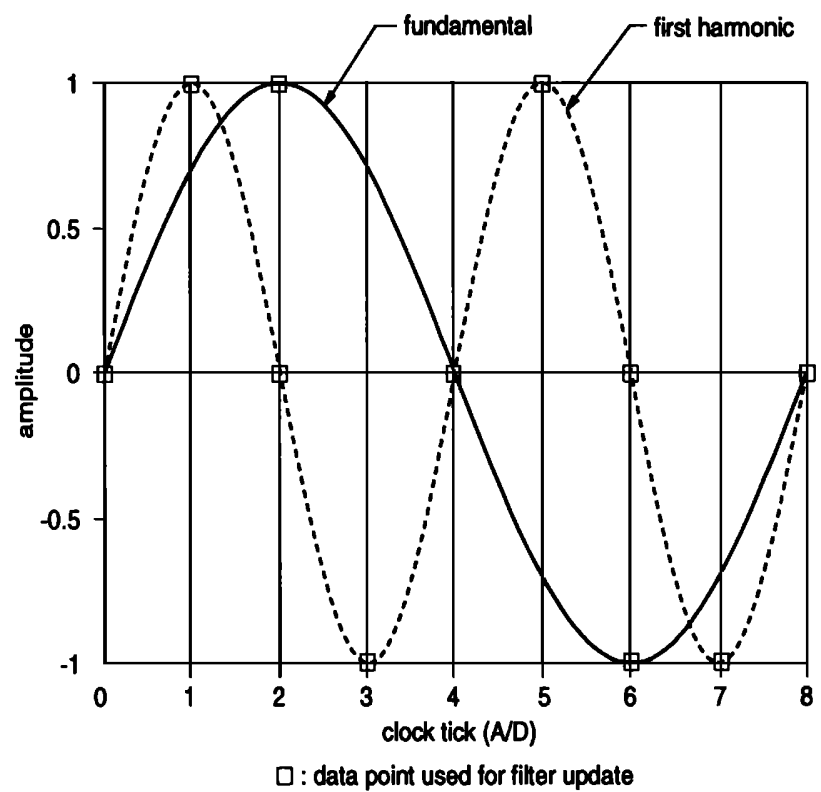

FIG. 3. Example of oversampling for synchronous sampling.

$\lambda_{\min }=\lambda_{\max }$ and the overall time constant is simply proportional to the trace of the input correlation matrix. The eigenvalues of the input correlation matrix effectively dictate the rate of convergence of each filter coefficient projected onto a set of orthogonal coordinates. Thus, disparity in the eigenvalues forces some filter coefficients to converge rapidly and others to converge more slowly. For a two-coefficient filter and a single-frequency disturbance, the most efficient and predictable convergence characteristics result when $N=4$.

These concepts can be extended to enhance the operation of the HLMS algorithm. If each of the two-coefficient adaptive FIR filters is implemented in parallel, the reference can be sampled at a rate such that typical oversampling techniques can be used for synchronous sampling of each independent reference generated to control each harmonic. As a simple example, assume that the fundamental and first harmonic are the only frequencies to be controlled. A schematic diagram of the two temporal waves is presented in Fig. 3, assuming that the first harmonic has been generated from the fundamental as outlined in Sec. II. The signals are marked with a square to indicate the data points used to update the adaptive FIR filter for each respective signal. The vertical lines indicate the clock ticks for each analog to digital (A/D) conversion. Thus, if the fundamental is sampled at eight times per period, then the first harmonic is effectively sampled at four times per period as illustrated in Fig. 3. By only computing the update for the parallel controller of the fundamental frequency at every other clock tick, synchronous sampling at four times per period can be implemented for the fundamental as well.

An additional benefit results from synchronous sampling at a rate of four samples per period of each harmonic to be controlled. As previously discussed by Clark and Gibbs (1994), if noise is present on the reference input, the system identification required for the filtered-x reference can be significantly distorted. At the recommended sampling rate, the distortion is minimum and thus the system identification pro- 


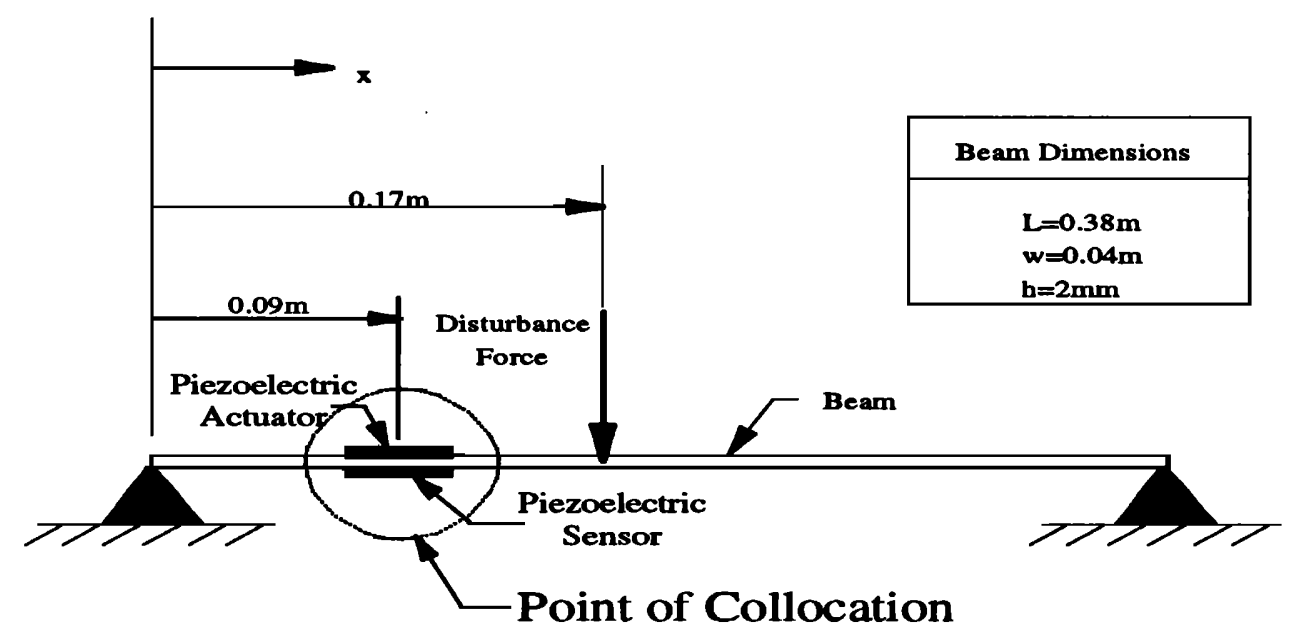

FIG. 4. Schematic of steel beam for simulation.

cess is optimal for the given variance of the noise present. However, at any other sampling rate, slower or faster, the noise significantly deteriorates the system identification.

\section{DISCUSSION OF TEST CASE}

The test structure chosen for the simulation was a simply supported beam since the structural response can be modeled analytically. The discrete time velocity response of the structure was obtained from a state-space formulation of the modal response of the system over the first five modes as outlined by Meirovitch (1990). For harmonic control, it makes no difference whether the output at the error sensor is a function of displacement, velocity, or acceleration. A zeroth-order hold was implemented in the model of the structural error response to accurately represent the dynamics introduced in the sampled-data system since the error signal must be sampled in discrete time. Five structural modes were sufficient for simulating the response of the beam since the frequency range of excitation was well below the resonant frequencies of the higher modes. Since future experiments will incorporate a shaker as the input disturbance and a piezoelectric actuator as the control, models of each respective input were obtained. The formulation of the models for the actuator and disturbance input can be found in a number of references (Burke and Hubbard, 1990; Clark et al., 1993; Clark, 1994). A piezoelectric sensor was also chosen for the simulation, and it was collocated with the control actuator.

A schematic of the beam is illustrated in Fig. 4 with all appropriate dimensions. Material properties of the beam were based upon that of steel with resonant frequencies listed in Table I. The point force disturbance was positioned $0.17 \mathrm{~m}$ from the left end of the beam and the piezoelectric actuator was centered at a coordinate $0.09 \mathrm{~m}$ from the same end as illustrated in Fig. 4. The piezoelectric actuator used in the simulations was rectangular and dimensions were selected to match the width of the beam: $40 \mathrm{~mm} \times 40 \mathrm{~mm} \times 0.19 \mathrm{~mm}$ thick. The dimensions of the collocated piezoelectric sensor were selected to match those of the actuator.
A single-frequency signal was generated as the input reference for the HLMS control algorithm, and the reference and the first four harmonics were implemented as the input point force disturbance. Hence the input disturbance includes four additional frequencies not present in the reference for the feedforward controller. The sampling rate of the controller was set at $1000 \mathrm{~Hz}$, approximately 20 times faster than the fundamental frequency to be controlled. As discussed earlier, the sampling rate must be approximately four times faster than the highest frequency to be controlled (which corresponds to the fourth harmonic at $250 \mathrm{~Hz}$ ) in the HLMS approach to approximate an LTI system.

To provide a basis of comparison and demonstrate the performance advantage gained with the proposed filter structure of the HLMS algorithm, the multifrequency, filtered-x LMS algorithm was implemented to control the structural response as well. The multifrequency input disturbance was used as the reference signal for the feedforward control algorithm. Thus, an adaptive FIR filter with at least ten coefficients is required to generate the control signal for the actuator. The comparison between the two control approaches serves to demonstrate the advantages associated with the HLMS filter structure and the potential limitations imposed by the multifrequency, filtered-x LMS algorithm.

\section{RESULTS}

Four unique test cases are presented in this section. In the first three test cases, the multi-frequency, filtered-x LMS algorithm is used to generate the control signal. The disad-

TABLE I. Theoretical beam resonant frequencies, $f_{m n}$.

\begin{tabular}{cc}
\hline \hline Mode & $\begin{array}{c}\text { Theoretical } \\
\text { frequency } \\
(\mathrm{Hz})\end{array}$ \\
\hline$(1)$ & 31.1 \\
$(2)$ & 124.4 \\
$(3)$ & 279.9 \\
$(4)$ & 497.6 \\
$(5)$ & 777.5 \\
\hline
\end{tabular}



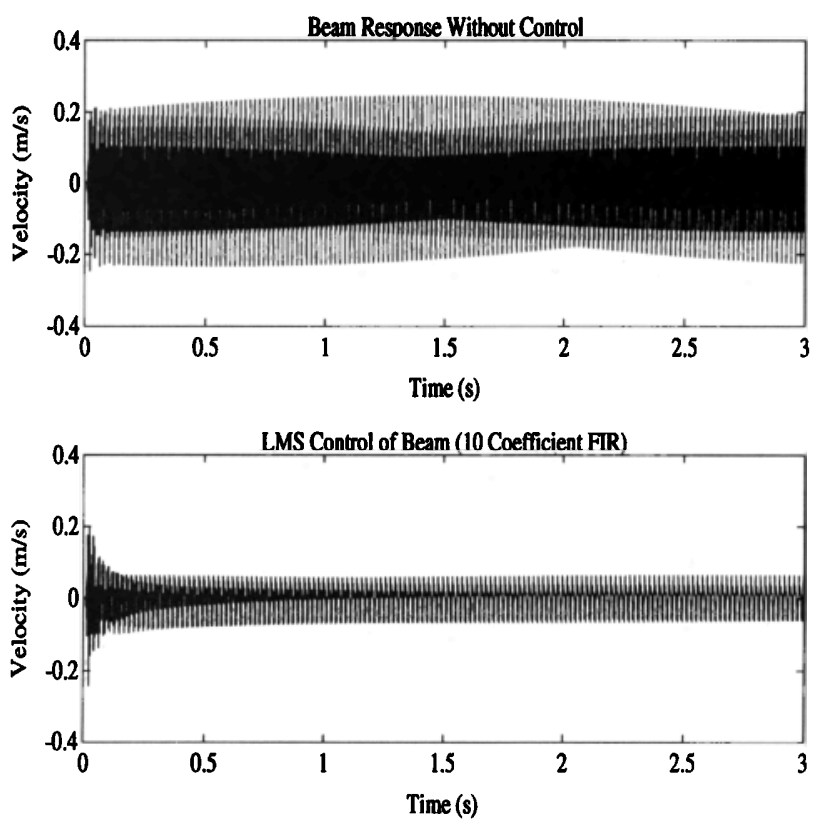

FIG. 5. Simulation multifrequency, filtered-x LMS algorithm with a tencoefficient FIR filter $(T=1 / 1000)$.

vantages associated with this approach are highlighted by example. In the final test case, the HLMS algorithm is used to generate the same required control signal. In all of the results presented, one should recognize that the harmonic time trace might appear somewhat distorted. The distortion results from displaying only a limited number of sampleddata points over the time-trace as opposed to every sample point.

\section{A. The multifrequency, filtered-x LMS algorithm}

The time-dependent, uncontrolled response of the structure at the selected error sensor is illustrated in the top portion of Fig. 5. A ten-coefficient adaptive FIR was implemented to generate the control signal based upon the multifrequency filtered-x LMS algorithm, and the result of this control is presented in the lower portion of Fig. 5. The sampling rate was set at $1000 \mathrm{~Hz}$, which is four times that of the highest harmonic present at $250 \mathrm{~Hz}$. The convergence parameter was set for the optimal rate of convergence; however, as observed, the error oscillates about the optimal'solution. This oscillation is a direct result of the TV terms discussed previously by Glover (1977).

The adaptive weights converge to a dynamic solution that instantaneously satisfies the convergence criterion, but does not converge to the optimal Wiener-Hopf solution. The adaptation of the FIR filter for this test case is presented in Fig. 6 and, as illustrated, the weights converge to a dynamic solution that oscillates about a mean. The mean value of the converged filter coefficients is presented in Table II, and the weights are compared to those obtained by solving for the optimal Wiener-Hopf solution. The mean value of the weights differs significantly from the optimal weights upon converging. This behavior is a direct result of the timevarying part of the solution. As discussed earlier, and as outlined by Glover (1977) in the active control of interference

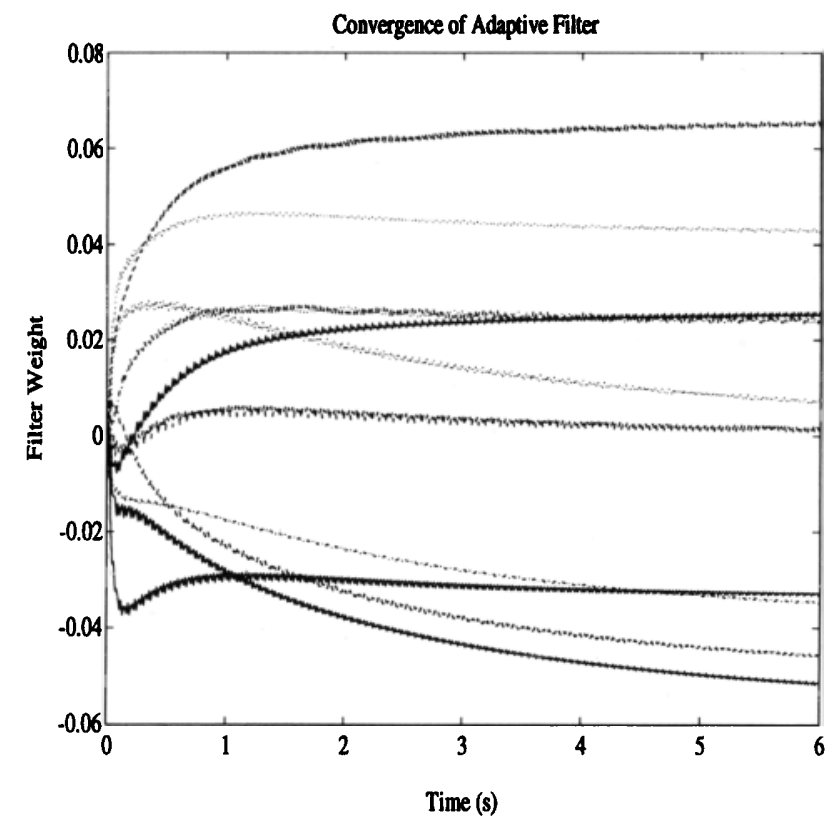

FIG. 6. Convergence of adaptive FIR filter $(T=1 / 1000)$.

noise, the number of coefficients included in the adaptive filter can be increased to reduce the effect of the TV terms in Eq. (5).

In the second test case, the sampling rate was reduced to $500 \mathrm{~Hz}$, which is twice the frequency of the highest harmonic (i.e., the Nyquist rate). Reviewing Eq. (5), one observes that $\beta(\omega(n-m) T / 2, I)$ and $\beta(\omega(n+m) T / 2, I)$ are identically zero. Thus, for this special higher harmonic control case, the system is LTI and the algorithm will converge to the optimal solution. The results from the simulation are presented in Fig. 7. The convergence parameter was adjusted for optimal rate of convergence, but as indicated in the results, after $3 \mathrm{~s}$ of adaptation, the minimum mean square error has not been achieved. All harmonics but the fundamental have essentially been eliminated from the error signal. The convergence of the filter with respect to the fundamental frequency of excitation takes place at a much slower rate because of the disparity in the eigenvalues of the input correlation matrix, which is related to the fastest and slowest time scales of the system as discussed by Haykin (1991) as well as Sievers and von Flotow (1990).

To increase the rate and level of convergence, the order

TABLE II. Comparison of optimal Wiener solution to LMS Solution for ten-coefficient FIR filter.

\begin{tabular}{ccc}
\hline \hline Coefficient & Adaptive result & Optimal Wiener \\
\hline$W_{0}$ & -0.05134 & -1.6105 \\
$W_{1}$ & 0.06516 & 7.3425 \\
$W_{2}$ & 0.04294 & -18.755 \\
$W_{3}$ & -0.03463 & 33.232 \\
$W_{4}$ & -0.03282 & -43.975 \\
$W_{5}$ & 0.001541 & 44.712 \\
$W_{6}$ & 0.007192 & -35.031 \\
$W_{7}$ & 0.02423 & 20.636 \\
$W_{8}$ & 0.02541 & -8.3069 \\
$W_{9}$ & -0.04558 & 1.8445 \\
\hline \hline
\end{tabular}



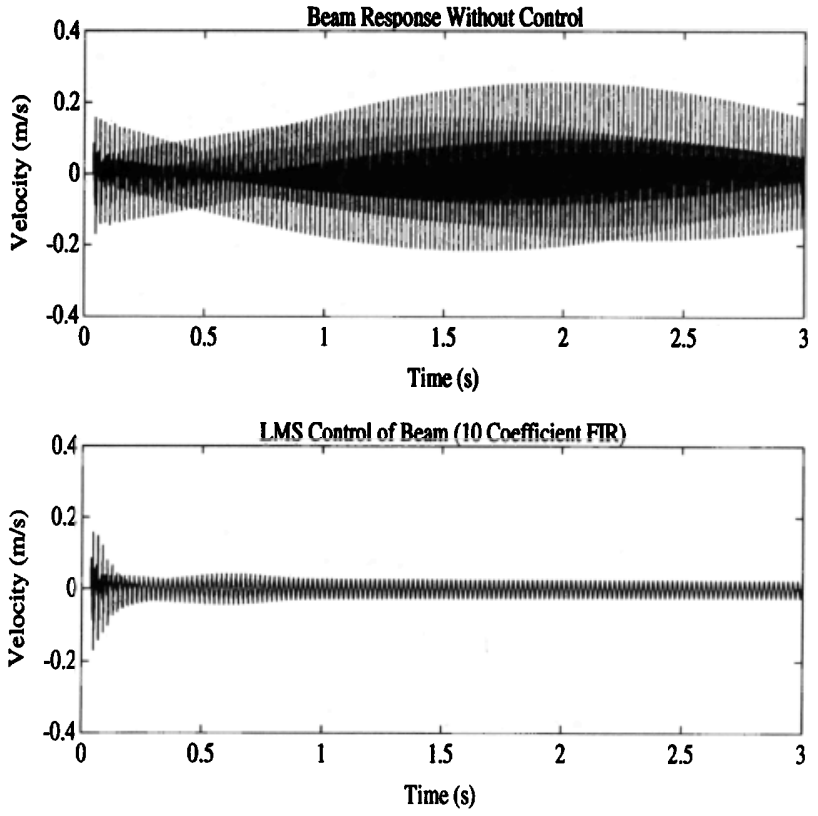

FIG. 7. Simulation multifrequency, filtered-x LMS algorithm with a tencoefficient FIR filter $(T=1 / 500)$.

of the adaptive filter was increased by a factor of 2 from 10 to 20 , and the sampling rate was doubled from 500 to 1000 Hz. Multiplying both the sampling rate and the order of the adaptive filter by the same integer guarantees that an LTI system will result since $\beta(\omega(n-m) T / 2, I)$ and $\beta(\omega(n$ $+m) T / 2, I)$ are held fixed at zero. The reader should be reminded that this scenario is unique to the higher-harmonic control problem and, in the general multifrequency control problem, this relationship will not hold. The results from this simulation are presented in Fig. 8. The error signal is observed to converge to nearly zero in approximately $3 \mathrm{~s}$ and, as in the previous test case, the convergence of the funda-
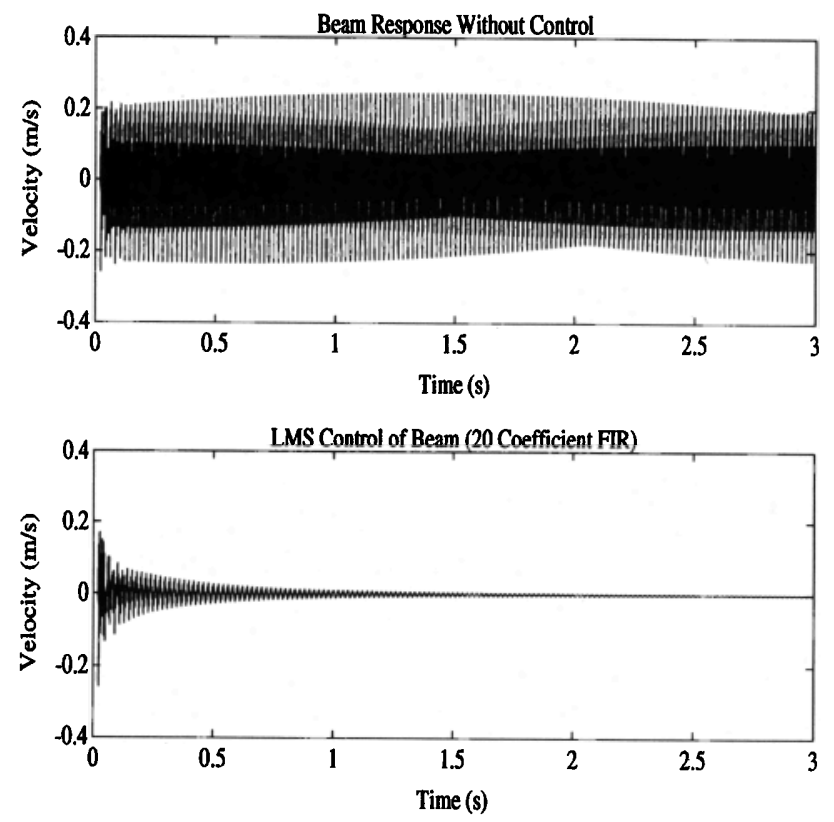

FIG. 8. Simulation multifrequency, filtered-x LMS algorithm with a 20coefficient FIR filter $(T=1 / 1000)$.
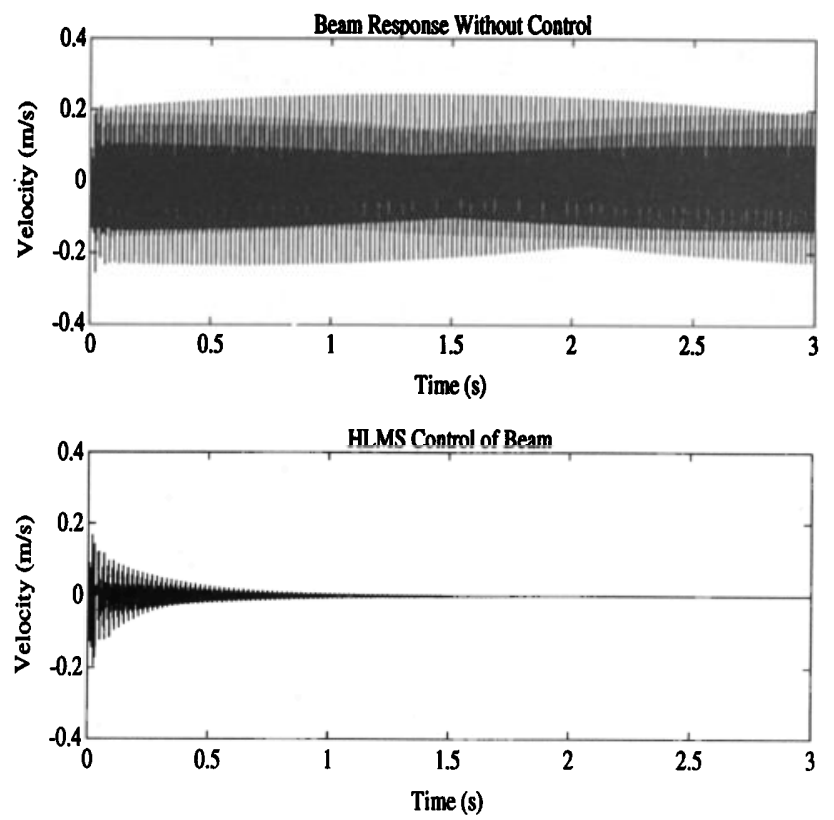

FIG. 9. Simulation of HLMS algorithm with five parallel two-coefficient FIR filters $(T=1 / 1000)$.

mental frequency dictates the overall rate of convergence of the system. Thus, one can certainly achieve control with the multifrequency, filtered-x LMS algorithm, but the order of the adaptive filter must be increased significantly to achieve a reasonable rate of convergence, and the reference input to the adaptive filter must contain the fundamental and all associated harmonics.

\section{B. The higher-harmonic, least-mean squares (HLMS) algorithm}

To circumvent this problem, the filter structure proposed for the HLMS algorithm was implemented to generate the desired control signal. In this case, separate two-coefficient adaptive filters are implemented in parallel with unique reference signals generated internally in software as discussed earlier and illustrated in Fig. 2. Individual convergence parameters were optimized for each parallel implementation of the LMS algorithm. Hence, the convergence of the adaptive filters for each frequency is effectively performed on the same time scale, overcoming limitations on rate of convergence imposed by the multifrequency, filtered-x LMS algorithm. The results from the simulation for this control scenario are presented in Fig. 9. As illustrated, the solution is observed to converge to zero in approximately $1 \mathrm{~s}$. No oscillation occurs about the optimal solution upon converging since the TV terms were effectively eliminated by implementing oversampling techniques such that roughly four samples per period were utilized in the adaptation of each parallel set of two-coefficient FIR filters. However, regardless of the chosen sampling rate, the HLMS algorithm will always converge to the optimal Wiener-Hopf solution since each frequency is controlled independently. Thus the reference signal to each parallel adaptive filter is correlated only 
with a single frequency present in the error, causing the contribution of the time-varying terms to diminish with successive iterations.

\section{CONCLUSIONS}

A novel filter structure for higher harmonic control was developed and designated the HLMS algorithm. The adaptation of this feedforward controller was compared to that of the standard multifrequency, filtered-x LMS algorithm. The HLMS algorithm offers significant performance advantages over the multifrequency, filtered-x LMS algorithm since oversampling techniques can be employed to minimize the response of the time-varying terms in the adaptation process, and the convergence parameters can be optimized for each frequency to be controlled. In addition, the minimum number of adaptive filter coefficients required for a desirable rate and level of convergence in the mean-squared error is guaranteed with the HLMS implementation; however, larger filter structures are required with the multifrequency, filtered-x LMS algorithm to achieve comparable results.

In the multifrequency, filtered-x LMS algorithm, the time-varying terms cause the adaptive filter to converge to a dynamic solution, and thus the weights oscillate about some mean value that does not in general correspond to the optimal Wiener-Hopf solution for arbitrary sampling rates. The effect of the time-varying terms can be reduced by increasing the order of the adaptive filter, at the obvious expense of storage requirements and system throughput in practical applications. In the case of higher-harmonic control, if the sampling rate is selected at twice the frequency of the highest harmonic to be controlled, then an LTI system results, and the algorithm converges to the optimal Wiener-Hopf solution. However, adaptation is slow since the magnitude of the convergence parameter is limited by the dynamics of the fastest poles of the linear system. To increase the rate of convergence, larger adaptive filters can be implemented, at the expense of memory storage and system throughput.

With the HLMS algorithm, oversampling techniques are implemented to assure that the time-varying portion of the adaptation is minimized or totally eliminated. Thus, one need not increase the order of the filter to achieve optimal performance in both rate of convergence and level of attenuation. In addition, since the convergence parameters can be uniquely optimized for each frequency present in the reference, the optimal rate of convergence is guaranteed. Results from this study demonstrate the novelty of the proposed filter structure and highlight the potential problems associated with the multifrequency, filtered-x LMS algorithm.

\section{APPENDIX: Z-TRANSFORM OF ADAPTIVE FILTER}

The $z$ transform of the adaptive filter for a multifrequency reference input is derived in this section for the SISO system illustrated in Fig. 1. The reference input is expressed in discrete time as a function of the fundamental and $M-1$ harmonics:

$$
x(k)=\sum_{m=1}^{M} \cos (\omega m k T),
$$

where $\omega$ is the circular frequency of the fundamental, $m-1$ is the order of the harmonic, $k$ is the time step, and $T$ is the sample period. In the active control of a linear system, the output of the system transfer functions are summed at the error sensor. Thus, the filtered-x adaptive LMS algorithm is expressed as follows (Widrow and Stearns, 1985):

$$
w_{i}(k+1)=w_{i}(k)-\mu e(k) \hat{x}(k-i),
$$

where $w_{i}(k)$ is the $i$ th coefficient of the adaptive filter at the $k$ th time step, $\mu$ is the convergence parameter, and $\hat{x}(k)$ is the filtered-x signal at the $k$ th time step. The modulus and phase of the transfer function between the control actuator and the error sensor $\left(H_{c e}\right)$ at each frequency can be represented as follows:

$$
H_{c e}(\exp (j \omega m T))=H_{m} \exp \left(j \Phi_{m}\right),
$$

where $H_{m}$ is the magnitude and $\Phi_{m}$ is the phase. Thus the filtered-x signal can be obtained,

$$
\hat{x}(k)=\sum_{m=1}^{M} H_{m} \cos \left(\omega m k T+\Phi_{m}\right),
$$

and hence can be expressed as

$$
\begin{aligned}
\hat{x}(k-i)= & \sum_{m=1}^{M} \frac{H_{m}}{2}\left\{\exp (j \omega m k T) \exp \left[j\left(\Phi_{m}-\omega m T i\right)\right]\right. \\
& \left.+\exp (-j \omega m k T) \exp \left[-j\left(\Phi_{m}-\omega m T i\right)\right]\right\}
\end{aligned}
$$

Taking the $z$ transform of Eq. (A2), one obtains

$$
Z\left\{w_{i}(k+1)\right\}=Z\left\{w_{i}(k)\right\}-\mu Z\{e(k) \hat{x}(k-i)\},
$$

where

$$
\begin{aligned}
Z\{e(k) \hat{x}(k-i)\}= & \sum_{m=1}^{M} \frac{H_{m}}{2}[E(z \exp (-j \omega m T)) \\
& \times\left\{\exp \left[j\left(\Phi_{m}-\omega m T i\right)\right]\right\} \\
& +E(z \exp (j \omega m T)) \\
& \left.\times\left\{\exp \left[-j\left(\Phi_{m}-\omega m T i\right)\right]\right\}\right]
\end{aligned}
$$

and $E(z)$ is the $z$ transform of $e(t)$. The $z$ transform of the adaptive filter is obtained as follows:

$$
\begin{aligned}
W_{i}(z)= & -\frac{\mu}{2} U(z) \sum_{m=1}^{M} H_{m}[E(z \exp (-j \omega m T)) \\
& \times\left\{\exp \left[j\left(\Phi_{m}-\omega m T i\right)\right]\right\} \\
& \left.+E(z \exp (j \omega m T))\left\{\exp \left[-j\left(\Phi_{m}-\omega m T i\right)\right]\right\}\right]
\end{aligned}
$$

where

$$
U(z)=1 /(z-1) .
$$

Now, the output of the adaptive filter is formed as a function of the reference input and the $I$ coefficients of the filter: 


$$
\begin{aligned}
y(k)= & \sum_{m=1}^{M} \sum_{i=0}^{I-1} w_{i}(k) \cos [\omega m(k-1) T] \\
= & \frac{1}{2} \sum_{m=1}^{M} \sum_{i=0}^{I-1} w_{i}(k)[\exp (j \omega m k T) \exp (-j \omega m i T) \\
& +\exp (-j \omega m k T) \exp (j \omega m i T)]
\end{aligned}
$$

Taking the $z$ transform of the output,

$$
\begin{aligned}
Y(z)= & \frac{1}{2} \sum_{m=1}^{M} \sum_{i=0}^{I-1}\left[W_{i}(z \exp (-j \omega m T)) e^{-j \omega m i T}\right. \\
& \left.+W_{i}(z \exp (j \omega m T)) \exp (j \omega m i T)\right] .
\end{aligned}
$$

Substituting Eq. (A8) into the above expression yields

$$
\begin{aligned}
Y(z)= & -\frac{\mu}{4} \sum_{m=1}^{M} \sum_{n=1}^{M} \sum_{i=0}^{I-1} H_{m}\left[U ( z \operatorname { e x p } ( - j \omega n T ) ) \operatorname { e x p } ( - j \omega n i T ) \left\{\exp \left[j\left(\Phi_{m}-\omega m T i\right)\right] E(z \exp (-j \omega m T) \exp (-j \omega n T))\right.\right. \\
& \left.+\exp \left[-j\left(\Phi_{m}-\omega m T i\right)\right] E(z \exp (j \omega m T) \exp (-j \omega n T))\right\}+\left[U ( z \operatorname { e x p } ( j \omega n T ) ) \operatorname { e x p } ( j \omega n T i ) \left\{\exp \left[j\left(\Phi_{m}-\omega m T i\right)\right]\right.\right. \\
& \left.\left.\times E(z \exp (-j \omega m T) \exp (j \omega n T))+\exp \left[-j\left(\Phi_{m}-\omega m T i\right)\right] E(z \exp (j \omega m T) \exp (j \omega n T))\right\}\right] .
\end{aligned}
$$

As discussed by Glover (1977), the summation over the $I$ adaptive filter coefficients can be replaced in the above expression since

$$
\sum_{i=0}^{I-1} \exp ( \pm j 2 \omega m T i)=\frac{1-\exp ( \pm j 2 \omega m T I)}{1-\exp ( \pm j 2 \omega m T)}=\exp [ \pm j 2 \omega m T(I-1)] \frac{\sin (\omega m T I)}{\sin (\omega m T)}=\exp [ \pm j 2 \omega m T(I-1)] \beta(\omega m T, I)
$$

Collecting terms, Eq. (A12) can be expressed as a function of the LTI and TV terms as

$$
\begin{aligned}
Y(z)= & -\frac{\mu I}{4} \sum_{m=1}^{M} H_{m} E(z)\left[U(z \exp (-j \omega m T)) \exp \left(-j \Phi_{m}\right)+U(z \exp (j \omega m T)) \exp \left(j \Phi_{m}\right)\right] \\
& -\frac{\mu}{4} \sum_{m=1}^{M} \sum_{\substack{n=1 \\
m \neq n}}^{M} H_{m}\left\{E(z \exp [-j \omega(n-m) T]) \exp [-j \omega(n-m) T(I-1)] \exp \left(-j \Phi_{m}\right) U(z \exp (-j \omega n T))\right. \\
& \left.+E(z \exp [j \omega(n-m) T]) \exp [j \omega(n-m) T(I-1)] \exp \left(j \Phi_{m}\right) U(z \exp (j \omega n T))\right\} \beta\left(\frac{\omega(m-n)}{2} T, I\right) \\
& -\frac{\mu}{4} \sum_{m=1}^{M} \sum_{n=1}^{M} H_{m}\left\{E(z \exp [-j \omega(n+m) T]) \exp [-j \omega(n+m) T(I-1)] \exp \left(j \Phi_{m}\right) U[z \exp (-j \omega n T)]\right. \\
& \left.+E(z \exp [j \omega(n+m) T]) \exp [j \omega(n+m) T(I-1)] \exp \left(-j \Phi_{m}\right) U[z \exp (j \omega n T)]\right\} \beta\left(\frac{\omega(m+n)}{2} T, I\right) .
\end{aligned}
$$

The first summation in the above equation represents the LTI term, and the remaining two summations represent the TV terms. As indicated, adding multiple frequencies to the reference input complicates the TV terms. However, as noted by Glover (1977), the contribution of the TV terms can be minimized as the ratio of $\beta(\omega(m \pm n) T / 2, I) / I$ approaches zero. Thus one must typically increase the order of the adaptive filter to minimize the effect of the TV terms on the solution.

Burke, S. E. and Hubbard, J. E. (1990). "Spatial filtering concepts in distributed parameter control," J. Dynamic Systems Measurement Control 112, 565-573.
Clark, R. L. (1994). "A hybrid, autonomous control algorithm," accepted to J. Dynamic Systems Measurement Control.

Clark, R. L. and Gibbs, G. P. (1994). "Implications of noise on the reference signal in feedforward control of harmonic disturbances," accepted to $J$. Acoust. Soc. Am.

Clark, R. L., Burdisso, R. A., and Fuller, C. R. (1993). "Design approaches for shaping polyvinylidene fluoride sensors in active structural acoustic control," J. Intelligent Mater. Systems Structures 4, 354-365.

Elliott, S. J., Strothers, I. M., and Nelson, P. A. (1987). "A multiple error LMS algorithm and its application to the active control of sound and vibration," IEEE Trans. Acoust. Speech Signal Process. ASSP-35, 14231434.

Glover, J. R. (1977). "Adaptive noise canceling applied to sinusoidal interferences,” IEEE Trans. Acoust. Speech Signal Process. ASSP-25, 484490. 
Griffiths, L. J. (1975). "Rapid measurement of digital instantaneous frequency," IEEE Trans. Acoust. Speech Signal Process. ASSP-23, 207-222. Hall, S. R., and Wereley, N. M. (1989). "Linear control issues in the higher harmonic control of helicopter vibrations," Proceedings of the 45th Annual Forum of the American Helicopter Society, Boston, MA, pp. 955971.

Haykin, S. (1991). Adaptive Filter Theory (Prentice-Hall, Englewood Cliffs, NJ), pp. 275-284.

Meirovitch, L. (1990). Dynamics and Control of Structures (Wiley, New York).
Shaw, J., and Albion, N. (1981). "Active control of the helicopter rotor for vibration reduction," J. Am. Helicopter Soc. 26(3), 32-39.

Shaw, J., Albion, N., Hanker Jr., E., and Teal, R. (1989). "Higher harmonic control: Wind tunnel demonstration of fully effective vibratory hub force suppression," J. Am. Helicopter Soc. 34(1), 14-25.

Sievers, L. A., and von Flotow, A. H. (1990). "Comparison and extensions of control methods for narrowband disturbance rejection," in Proceedings of the ASME Winter Annual Meeting, Dallas, TX (ASME, New York).

Widrow, B., and Stearns, S. D. (1985). Adaptive Signal Processing (Prentice-Hall, Englewood Cliffs, NJ). 